\title{
Projection de la demande de lits de soins intensifs durant l'épidémie de COVID-19 au Canada
}

\author{
Affan Shoukat PhD, Chad R. Wells PhD, Joanne M. Langley MD, Burton H. Singer PhD, Alison P. Galvani PhD, \\ Seyed M. Moghadas PhD
}

E Citation : CMAJ 2020 May 11;192:E489-96. doi : 10.1503/cmaj.200457-f; diffusion hâtive le 8 avril 2020

Voir la version anglaise de l'article ici : www.cmaj.ca/lookup/doi/10.1503/cmaj.200457; voir l'éditorial connexe (en anglais et français) ici : www.cmaj.ca/lookup/doi/10.1503/cmaj.200606

\section{RÉSUMÉ}

CONTEXTE : La hausse des cas de maladie à coronavirus 2019 (COVID-19) au Canada peut créer une forte demande de soins hospitaliers et de soins intensifs. Nous avons évalué la mesure dans laquelle l'isolement volontaire des personnes présentant des symptômes légers retarde le sommet épidémique et réduit la demande de soins dans chaque province canadienne.

MÉTHODES : Nous avons conçu un modèle de calcul et fait des simulations de la propagation de la COVID-19 dans chaque province. À partir des estimations des caractéristiques de la COVID19 , nous avons évalué la demande de lits d'hôpital et de lits de soins intensifs en l'absence d'isolement volontaire en supposant une moyenne de 2,5 cas secondaires, et avons comparé des scénarios en faisant varier le taux d'isolement volontaire des cas légers 24 heures après l'apparition des symptômes.

RÉSULTATS : En l'absence d'isolement volontaire, l'épidémie atteindrait son sommet dans la première moitié de juin, et il faudrait en moyenne 569 jours-lits de soins intensifs par 10000 habitants. Avec un taux d'isolement volontaire de $20 \%$, l'atteinte du sommet serait repoussée de 2 à 4 semaines, et la demande de lits diminuerait de $23,5 \%$; avec un taux de $40 \%$, le sommet serait repoussé de 2 à 4 semaines supplémen- taires, et la demande de lits connaîtrait une baisse de 53,6\%. En fixant le taux d'occupation actuel des lits de soins intensifs à plus de $80 \%$ et le taux d'isolement volontaire à $40 \%$, la demande de lits demeure supérieure au nombre de lits disponibles.

INTERPRÉTATION : Au sommet de l'épidémie de COVID-19 au Canada, la demande de lits de soins intensifs excédera le nombre total de lits disponibles, même avec un taux d'isolement volontaire de $40 \%$. Nos résultats montrent que la situation sera difficile pour le système de santé et que l'isolement volontaire pourrait réduire la demande de soins hospitaliers et de soins intensifs.
D epuis l'identification en décembre 2019 du coronavirus du syndrome respiratoire aigu sévère 2 (SRAS-CoV-2) en Chine, on a répertorié plus de 850000 cas confirmés de maladie à coronavirus 2019 (COVID-19) dans 180 pays, dont le Canada ${ }^{1}$. En date du 31 mars 2020, on compte 8591 cas confirmés au pays, dont une partie est due à la transmission locale qui sévit dans plusieurs régions. Pour limiter la transmission, les autorités de santé publique fédérales préconisent l'éloignement social et l'isolement volontaire des personnes potentiellement exposées et des personnes qui présentent des symptômes assez légers pour ne pas aller à l'hôpital.

Les premières éclosions et les observations des pays touchés indiquent que les patients atteints font exploser la demande de soins hospitaliers et de soins intensifs ${ }^{2-7}$. Les principales mesures de santé publique visant à atténuer cette montée sont la pratique de l'éloignement social volontaire tel que l'isolement volontaire, l'évitement des rassemblements de masse et l'amélioration des habitudes d'hygiène. D'ici à ce qu'il y ait un vaccin ou des traitements contre la maladie, on recourt à ces interventions non pharmacologiques efficaces afin de réduire l'incidence des nouvelles infections et ainsi de les étaler dans le temps, c'est-à-dire « aplatir la courbe », comme on l'entend souvent. L'objectif est de ne pas surcharger les systèmes de santé ${ }^{7}$, puisqu'ils ont une capacité limitée. Bien que la stratégie s'inscrive dans un effort mondial de lutte contre la pandémie, on ne s'attend pas à ce qu'elle endigue la COVID-19.

Un point qui inquiète particulièrement la santé publique dans son travail de planification est l'utilisation de ressources médicales pour traiter les patients qui nécessitent des soins intensifs ${ }^{8}$. Prenons 
l'exemple de Winnipeg (Manitoba) : au plus fort de la première vague de propagation pandémique de la grippe A (H1N1), en juin 2009, les services de soins intensifs étaient intensément sollicités et fonctionnaient au maximum de leur capacité ${ }^{9,10}$. Au sommet de la deuxième vague, l'usage de la ventilation invasive était $14 \%$ plus élevé qu'en moyenne, et $50 \%$ de la hausse était attribuable à la $\mathrm{H} 1 \mathrm{~N}^{8}$. Comme le taux d'occupation des lits de soins intensifs était quasi maximal avant même la crise de santé publique ${ }^{11}$ et varie énormément d'une région à l'autre ${ }^{12}$, il est absolument essentiel d'évaluer la demande pour ces ressources dans un scénario d'épidémie de COVID-19.

Nous voulions donc prévoir la date du sommet épidémique et la demande de lits de soins intensifs associée dans les différentes provinces canadiennes.

\section{Méthodes}

Nous avons conçu un modèle à base d'agents intégrant l'âge, et l'avons paramétré selon la structure par âge de chaque province et les premières estimations des caractéristiques de la COVID-19. Dans nos analyses de scénarios, nous avons considéré l'isolement volontaire des cas légers comme une mesure de contrôle et évalué son effet sur la courbe épidémique et les besoins en soins intensifs. Puis nous avons fait des simulations en attribuant au nombre moyen de cas secondaires, aussi appelé le taux de reproduction de base $\left(R_{0}\right)$, la valeur de 2 et de $2,5^{13,14}$.

\section{Structure du modèle}

Nous avons conçu un modèle informatique stochastique à base d'agents stratifiés en groupes d'âge pour représenter la transmission de la COVID-19. Ce modèle fait intervenir des agents autonomes (les membres d'une population d'humains) et simule leurs interactions dans un environnement virtuel restreint. Il intègre les différents statuts infectieux individuels : susceptible; atteint et en période d'incubation; atteint et présentant une maladie légère, grave ou critique; rétabli; et décédé (figure 1). Pour chaque province, nous avons stratifié la population en 5 groupes d'âge $-0-4$ ans, 5-19 ans, 20-49 ans, 50-64 ans et $\geq 65$ ans - à partir des données du dernier recensement du Canada ${ }^{15}$. Puis nous avons simulé la transmission dans les groupes et entre eux à l'aide d'un réseau de contacts établi empiriquement ${ }^{16}$. C'est-à-dire que nous avons ventilé le nombre quotidien de contacts de chaque personne donnée par une distribution binomiale négative selon l'âge (tableau A1 de l'annexe 1 (en anglais), au www.cmaj.ca/lookup/suppl/ doi:10.1503/cmaj.200457/-/DC1) en appliquant une matrice des contacts en région urbaine et densément peuplée.

\section{Propagation de la maladie}

Nous avons reconstitué la transmission lors de contacts entre une personne susceptible et une personne symptomatique par une approche probabiliste, en utilisant la probabilité de transmission quotidienne déterminée par calage du modèle (tableau 1 ). Nous avons obtenu une période d'incubation moyenne de 5,2 jours ${ }^{13,18}$, suivie d'une phase symptomatique ayant une période de contagiosité moyenne de 4,6 jours. Nos calculs reposent sur l'intervalle de série (le délai entre l'apparition des symptômes chez le cas index et l'apparition des symptômes chez un cas secondaire) moyen, estimé à 7,5 jours $^{13}$. Le risque de développer une maladie légère, grave ou critique varie selon l'âge ${ }^{7,19}$. Nous avons aussi supposé que l'infectivité relative est $50 \%$ plus faible pour les cas légers par rapport aux cas graves ${ }^{17}$.

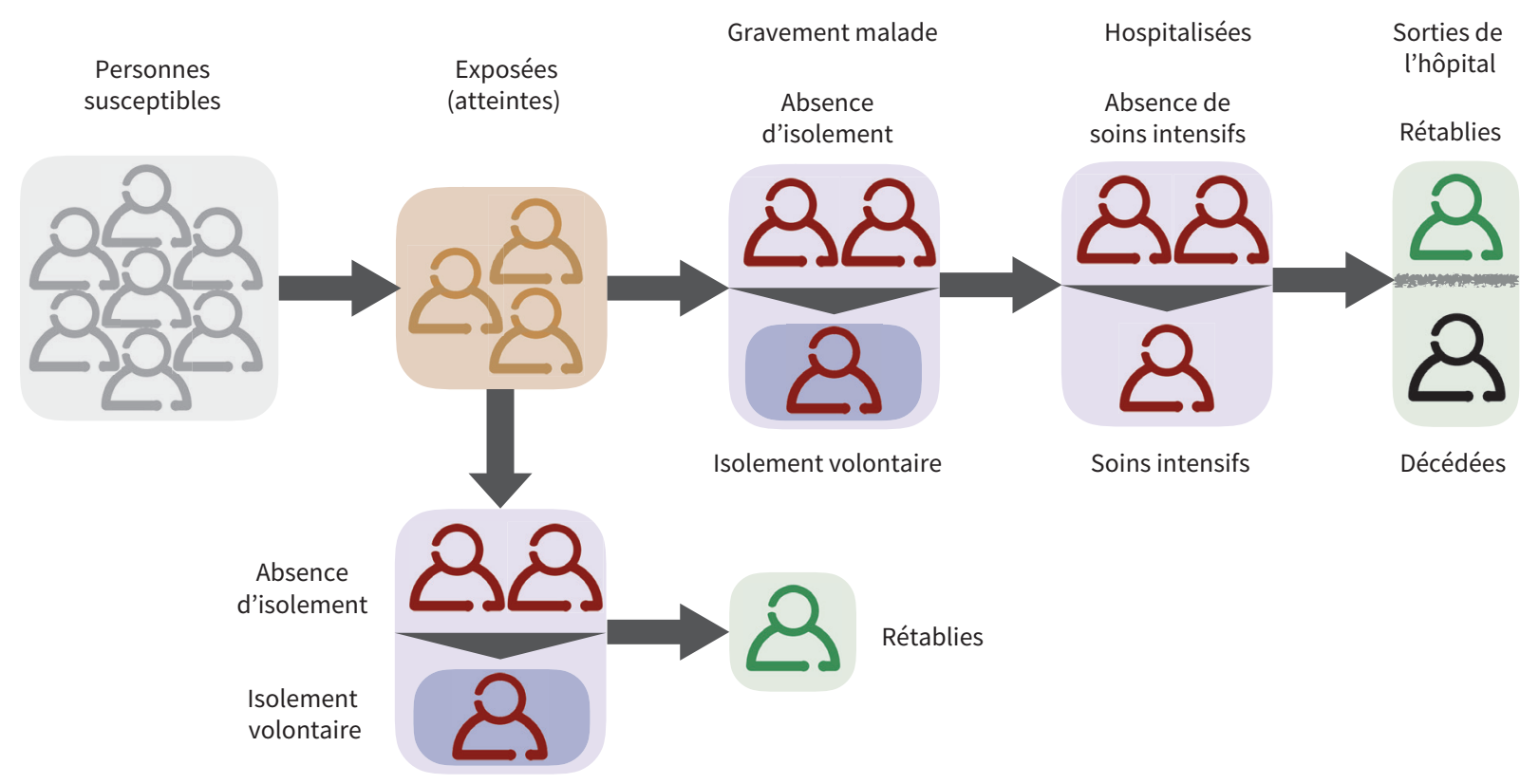

Légèrement malade

Figure 1. Schéma du modèle, représentant l'évolution naturelle de la maladie, l'adoption de l'isolement volontaire et le recours à l'hospitalisation. 


\section{Effets de l'infection}

Nous avons estimé que les personnes ayant des symptômes légers allaient se rétablir sans hospitalisation et se mettraient possiblement en isolement volontaire. L'hospitalisation et l'admission aux soins intensifs étaient seulement des options pour les cas graves et critiques. Dans les cas d'hospitalisation, le délai moyen entre l'apparition des symptômes et l'admission en établissement était toujours entre 2 et 5 jours ${ }^{7,20}$. Nous avons déterminé que les patients hospitalisés feraient l'objet de mesures de lutte anti-infectieuse efficaces et ne transmettraient donc pas le virus. Les patients hospitalisés ailleurs qu'aux soins intensifs ont occupé un lit en moyenne 11,5 jours avant de se rétablir ${ }^{7,20}$, et leur risque d'admission aux soins intensifs était corrélé à leur âge. Le temps moyen d'occupation d'un lit de soins intensifs avant rétablissement était de 14,4 jours?

\section{Isolement volontaire}

Nous avons présumé que les personnes symptomatiques qui se mettent en isolement volontaire le restent jusqu'à la fin de l'infection. Dans notre modèle, l'isolement volontaire correspond à un maximum de 3 contacts quotidiens. Comme nous n'avions pas de données sur la proportion des personnes symptomatiques qui décident de s'isoler, nous l'avons fait varier entre $0 \%$ et $80 \%$, et fait débuter l'isolement 24 heures après l'apparition des symptômes. Pour les cas graves, nous avons jugé que $80 \%$ s'isolent dans les 24 heures suivant l'apparition des symptômes (avant hospitalisation). Nous avons choisi ce pourcentage en fonction d'estimations antérieures ${ }^{22}$, et en tenant compte de la gravité perçue de la maladie et de l'insistance que les autorités de santé publique mettent sur l'éloignement social et l'isolement.

\section{Capacité des soins intensifs}

Une étude transversale nationale publiée en 2015 estime que le Canada compte un total de 3170 lits de soins intensifs où l'occupant peut recevoir une ventilation invasive ${ }^{12}$. Pour évaluer la demande de lits d'hôpital et de soins intensifs au sommet de la courbe épidémique, nous avons calculé le nombre de lits de soins intensifs dans chaque province et estimé, avec un taux d'occupation réaliste de $80 \%$, combien seraient disponibles pour les patients atteints de la COVID-19.

\section{Tableau 1. Description des paramètres, et valeurs ou intervalles pour chaque groupe d'âge}

\begin{tabular}{|c|c|c|c|c|c|}
\hline \multirow[b]{2}{*}{ Description } & \multicolumn{4}{|c|}{ Groupe d'âge } & \multirow[b]{2}{*}{ Références } \\
\hline & $0-19$ ans & $20-49$ ans & $50-64$ ans & 65 ans et plus & \\
\hline \multicolumn{6}{|l|}{$\begin{array}{l}\text { Probabilité de transmission par contact, selon } \\
\text { le taux de reproduction }\left(R_{0}\right)\end{array}$} \\
\hline$R_{0}=2$ & 0,0356 & 0,0356 & 0,0356 & 0,0356 & \multirow[t]{2}{*}{ Li et coll. ${ }^{13} ;$ Wu et coll. ${ }^{14}$} \\
\hline$R_{0}=2,5$ & 0,0455 & 0,0455 & 0,0455 & 0,0455 & \\
\hline $\begin{array}{l}\text { Infectivité relative pour les cas légers par } \\
\text { rapport aux cas graves }\end{array}$ & 0,5 & 0,5 & 0,5 & 0,5 & Li et coll. ${ }^{17}$ \\
\hline $\begin{array}{l}\text { Durée moyenne de la période d'incubation } \\
\text { (jours) }\end{array}$ & $\begin{array}{l}\text { Log-N } \\
(5,2 ; 0,1)\end{array}$ & $\begin{array}{l}\log -\mathrm{N} \\
(5,2 ; 0,1)\end{array}$ & $\begin{array}{l}\log -\mathrm{N} \\
(5,2 ; 0,1)\end{array}$ & $\begin{array}{l}\text { Log-N } \\
(5,2 ; 0,1)\end{array}$ & $\begin{array}{l}\text { Li et coll. }{ }^{13} ; \text { Lauer et } \\
\text { coll. }^{18}\end{array}$ \\
\hline $\begin{array}{l}\text { Proportion de personnes présentant des } \\
\text { symptômes légers (selon le nombre de cas } \\
\text { déclarés dans chaque groupe d'âge) }\end{array}$ & 0,80 & 0,80 & 0,40 & 0,20 & $\begin{array}{l}\text { Moghadas et coll. }{ }^{7} \\
\text { OMS }^{19}\end{array}$ \\
\hline $\begin{array}{l}\text { Durée moyenne de la période infectieuse } \\
\text { (jours) }\end{array}$ & 4,6 & 4,6 & 4,6 & 4,6 & Estimée \\
\hline $\begin{array}{l}\text { Probabilité d'isolement volontaire après } \\
\text { l'apparition de symptômes graves }\end{array}$ & 0,80 & 0,80 & 0,80 & 0,80 & Présumée \\
\hline $\begin{array}{l}\text { Probabilité d'isolement volontaire après } \\
\text { l'apparition de symptômes légers }\end{array}$ & $0-0,80$ & $0-0,80$ & $0-0,80$ & $0-0,80$ & Variable \\
\hline $\begin{array}{l}\text { Temps écoulé entre l'apparition des } \\
\text { symptômes et l'isolement volontaire (jours) }\end{array}$ & 1 & 1 & 1 & 1 & Présumé \\
\hline $\begin{array}{l}\text { Temps moyen écoulé entre l'apparition des } \\
\text { symptômes et l'admission à l'hôpital (jours) }\end{array}$ & $\begin{array}{l}\text { Unif } \\
(2 ; 5)\end{array}$ & $\begin{array}{l}\text { Unif } \\
(2 ; 5)\end{array}$ & $\begin{array}{l}\text { Unif } \\
(2 ; 5)\end{array}$ & $\begin{array}{l}\text { Unif } \\
(2 ; 5)\end{array}$ & $\begin{array}{l}\text { Moghadas et coll. }{ }^{7} \\
\text { Sanche et coll. }{ }^{20}\end{array}$ \\
\hline $\begin{array}{l}\text { Proportion de patients présentant des symptômes } \\
\text { graves et nécessitant une hospitalisation en soins } \\
\text { intensifs ou non intensifs }\end{array}$ & $\begin{array}{c}\text { Unif } \\
(0,02 ; 0,03)\end{array}$ & $\begin{array}{c}\text { Unif } \\
(0,28 ; 0,34)\end{array}$ & $\begin{array}{c}\text { Unif } \\
(0,28 ; 0,34)\end{array}$ & $\begin{array}{c}\text { Unif } \\
(0,60 ; 0,68)\end{array}$ & $\begin{array}{l}\text { Estimée à partir de } \\
\text { OMS }^{19} \text { et de MIDAS }\end{array}$ \\
\hline $\begin{array}{l}\text { Proportion de personnes hospitalisées en } \\
\text { soins intensifs }\end{array}$ & $\begin{array}{c}\text { Unif } \\
(0,01 ; 0,013)\end{array}$ & $\begin{array}{c}\text { Unif } \\
(0,03 ; 0,05)\end{array}$ & $\begin{array}{l}\text { Unif } \\
(0,05 ; 0,2)\end{array}$ & $\begin{array}{l}\text { Unif } \\
(0,05 ; 0,15)\end{array}$ & $\begin{array}{l}\text { Estimée à partir de } \\
\text { MIDAS }^{2}\end{array}$ \\
\hline $\begin{array}{l}\text { Durée d'hospitalisation avant le rétablissement } \\
\text { (jours) }\end{array}$ & \multicolumn{4}{|c|}{ Gamma $(4,5 ; 2,75)$ tronquée entre 8 et 17} & $\begin{array}{l}\text { Moghadas et coll. } \\
\text { Sanche et coll. }\end{array}$ \\
\hline $\begin{array}{l}\text { Durée d'hospitalisation en soins intensifs avant } \\
\text { le rétablissement (jours) }\end{array}$ & \multicolumn{4}{|c|}{ Gamma $(4,5 ; 2,75)$ tronquée entre 10 et 19} & $\begin{array}{l}\text { Moghadas et coll. } \\
\text { Sanche et coll. }\end{array}$ \\
\hline
\end{tabular}




\section{Mise en œuvre du modèle}

Toujours pour évaluer la demande de lits par provinces, nous avons utilisé un taux de reproduction de base $\left(R_{0}\right)$ de 2,5 pour le scénario de référence, et de 2,0 pour un autre scénario ${ }^{13,14}$. Nous avons paramétré le modèle de calcul à partir des valeurs des distributions pertinentes et des plus récentes estimations des caractéristiques de la COVID-19 (tableau 1). Le modèle a été créé dans le langage de programmation Julia ${ }^{24}$. Nous avons lancé chaque simulation avec 5 cas symptomatiques par 10000 habitants en date du $1^{\text {er }}$ mars 2020 . Nous avons déterminé l'incidence d'infection et l'incidence d'hospitalisation quotidiennes en faisant la moyenne des résultats de 500 simulations indépendantes. Nous avons également estimé le nombre moyen de jours-lits de soins intensifs nécessaires durant l'épidémie à partir de la prévalence moyenne pour l'ensemble des simulations, en comptant le nombre de jours où les lits sont occupés. Le modèle est décrit à l'adresse https://github.com/ affans/covid19abm.jl.

\section{Approbation éthique}

Cette recherche utilisant seulement des données publiques, nous n'avons pas eu à demander une approbation éthique.

\section{Résultats}

Le nombre de lits de soins intensifs des provinces varie entre 0,63 et 1,85 par 10000 habitants. En supposant un taux d'occupation de $80 \%$, nous avons calculé que le nombre de lits disponibles pour traiter les patients atteints de la COVID-19 variait entre 0,13 et 0,37 par 10000 habitants (tableau 2).

Tableau 2. Projection des besoins en lits de soins intensifs ou non intensifs au sommet de la courbe, par 10000 habitants, selon divers scénarios d'isolement volontaire des cas légers dans chaque province*

\section{Besoins en lits d'hôpital au sommet de la courbe}

\begin{tabular}{|c|c|c|c|c|c|c|c|}
\hline \multirow{2}{*}{$\begin{array}{l}\text { Variables } \\
\text { Isolement } \\
\text { volontaire (\%) }\end{array}$} & \multicolumn{3}{|c|}{ Lits de soins non intensifs } & \multicolumn{3}{|c|}{ Lits de soins intensifs } & \multirow[t]{2}{*}{$\begin{array}{l}\text { intensifs par } \\
10000 \text { habitants }\end{array}$} \\
\hline & 0 & 20 & 40 & 0 & 20 & 40 & \\
\hline \multicolumn{8}{|l|}{ Province } \\
\hline $\begin{array}{l}\text { Colombie- } \\
\text { Britannique }\end{array}$ & $\begin{array}{c}\text { Moyenne : 80,2 } \\
\text { Médiane : } 83 \\
\text { (El : 72-92) }\end{array}$ & $\begin{array}{c}\text { Moyenne : 46,6 } \\
\text { Médiane : 48 } \\
\text { (El : 38-55) }\end{array}$ & $\begin{array}{l}\text { Moyenne : } 18,6 \\
\text { Médiane : } 19 \\
(\text { El : } 12-25)\end{array}$ & $\begin{array}{c}\text { Moyenne : 7,5 } \\
\text { Médiane : } 7 \\
(\text { El : 6-10) }\end{array}$ & $\begin{array}{c}\text { Moyenne : 4,2 } \\
\text { Médiane : } 4 \\
(\text { El : } 2-6)\end{array}$ & $\begin{array}{c}\text { Moyenne : 1,6 } \\
\text { Médiane : } 1 \\
\text { (El : 0-3) }\end{array}$ & $\begin{array}{l}\text { Existants : 0,63 } \\
\text { Disponibles : 0,13 }\end{array}$ \\
\hline Alberta & $\begin{array}{c}\text { Moyenne : } 81 \\
\text { Médiane : } 82 \\
\text { (El : 73-93) }\end{array}$ & $\begin{array}{c}\text { Moyenne : 46,9 } \\
\text { Médiane : 48 } \\
\text { (El : 39-56) }\end{array}$ & $\begin{array}{l}\text { Moyenne : } 18,9 \\
\text { Médiane : } 19 \\
\text { (El : 14-25) }\end{array}$ & $\begin{array}{c}\text { Moyenne : 7,5 } \\
\text { Médiane : } 7 \\
(\text { El :6-9,5) }\end{array}$ & $\begin{array}{c}\text { Moyenne : 4,3 } \\
\text { Médiane : } 4 \\
(\text { EI : 3-6) }\end{array}$ & $\begin{array}{c}\text { Moyenne : 1,6 } \\
\text { Médiane : } 1 \\
\text { (El : 0-2) }\end{array}$ & $\begin{array}{l}\text { Existants : 0,68 } \\
\text { Disponibles : } 0,14\end{array}$ \\
\hline Saskatchewan & $\begin{array}{c}\text { Moyenne : 81,1 } \\
\text { Médiane : } 83 \\
(\text { El : 72-92) }\end{array}$ & $\begin{array}{c}\text { Moyenne : 47,0 } \\
\text { Médiane : } 48 \\
\text { (El : 39-56) }\end{array}$ & $\begin{array}{l}\text { Moyenne : } 18,9 \\
\text { Médiane : } 19 \\
\text { (El : 13-25) }\end{array}$ & $\begin{array}{l}\text { Moyenne : 7,6 } \\
\text { Médiane : } 7 \\
\text { (El : 5-10) }\end{array}$ & $\begin{array}{c}\text { Moyenne : 4,3 } \\
\text { Médiane : } 4 \\
(\text { EI : 3-6) }\end{array}$ & $\begin{array}{c}\text { Moyenne : } 1,8 \\
\text { Médiane : } 1 \\
(\text { El : 0,5-3) }\end{array}$ & $\begin{array}{l}\text { Existants : 0,93 } \\
\text { Disponibles : 0,19 }\end{array}$ \\
\hline Manitoba & $\begin{array}{c}\text { Moyenne : } 81 \\
\text { Médiane : } 82 \\
(\text { El : 73-95) }\end{array}$ & $\begin{array}{c}\text { Moyenne : 46,9 } \\
\text { Médiane : 48 } \\
\text { (El : 39-56) }\end{array}$ & $\begin{array}{l}\text { Moyenne : } 18,8 \\
\text { Médiane : } 19 \\
\text { (El : 13-25) }\end{array}$ & $\begin{array}{l}\text { Moyenne : 7,6 } \\
\text { Médiane : } 7 \\
(\text { El : 5-10) }\end{array}$ & $\begin{array}{c}\text { Moyenne : 4,2 } \\
\text { Médiane : } 4 \\
(\text { EI : 2-6) }\end{array}$ & $\begin{array}{c}\text { Moyenne : 1,8 } \\
\text { Médiane : } 1 \\
\text { (El : 0-3) }\end{array}$ & $\begin{array}{l}\text { Existants : 0,69 } \\
\text { Disponibles : } 0,14\end{array}$ \\
\hline Ontario & $\begin{array}{c}\text { Moyenne : 80,7 } \\
\text { Médiane : } 83 \\
\text { (El : 72-92) }\end{array}$ & $\begin{array}{c}\text { Moyenne : 47,0 } \\
\text { Médiane : 48 } \\
\text { (El : 39-65) }\end{array}$ & $\begin{array}{c}\text { Moyenne : } 18,9 \\
\text { Médiane : } 19 \\
\text { (El : 14-25) }\end{array}$ & $\begin{array}{c}\text { Moyenne : 7,5 } \\
\text { Médiane : } 7 \\
(\text { El : 5-9,5) }\end{array}$ & $\begin{array}{c}\text { Moyenne : 4,2 } \\
\text { Médiane : } 4 \\
(\text { EI : 2-6) }\end{array}$ & $\begin{array}{c}\text { Moyenne : } 1,7 \\
\text { Médiane : } 1 \\
(\text { El : } 1-3)\end{array}$ & $\begin{array}{l}\text { Existants : 0,79 } \\
\text { Disponibles : } 0,16\end{array}$ \\
\hline Québec & $\begin{array}{l}\text { Moyenne : 81,1 } \\
\text { Médiane : } 83 \\
\text { (El : 72-92) }\end{array}$ & $\begin{array}{c}\text { Moyenne : 47,0 } \\
\text { Médiane : 48 } \\
\text { (El : 40-56) }\end{array}$ & $\begin{array}{c}\text { Moyenne : } 18,3 \\
\text { Médiane : } 19 \\
\text { (El : 11-26) }\end{array}$ & $\begin{array}{c}\text { Moyenne : 7,5 } \\
\text { Médiane : } 7 \\
\text { (EI:5-9) }\end{array}$ & $\begin{array}{c}\text { Moyenne : 4,2 } \\
\text { Médiane : } 4 \\
(\text { El : } 2-6)\end{array}$ & $\begin{array}{c}\text { Moyenne : } 1,7 \\
\text { Médiane : } 1 \\
\text { (EI : 1-2) }\end{array}$ & $\begin{array}{l}\text { Existants : } 1,05 \\
\text { Disponibles : } 0,21\end{array}$ \\
\hline $\begin{array}{l}\text { Nouveau- } \\
\text { Brunswick }\end{array}$ & $\begin{array}{c}\text { Moyenne : 80,2 } \\
\text { Médiane : } 82 \\
\text { (El : 72-93) }\end{array}$ & $\begin{array}{c}\text { Moyenne : 46,6 } \\
\text { Médiane : } 48 \\
\text { (El : 39-55) }\end{array}$ & $\begin{array}{l}\text { Moyenne : } 18,7 \\
\text { Médiane : } 19 \\
\text { (El : 12-25) }\end{array}$ & $\begin{array}{c}\text { Moyenne : 7,6 } \\
\text { Médiane : } 8 \\
\text { (El : 5,5-9) }\end{array}$ & $\begin{array}{c}\text { Moyenne : 4,2 } \\
\text { Médiane : } 4 \\
(\text { El : } 2-4)\end{array}$ & $\begin{array}{c}\text { Moyenne : } 1,7 \\
\text { Médiane : } 1 \\
\text { (El : } 1-3)\end{array}$ & $\begin{array}{l}\text { Existants : } 1,36 \\
\text { Disponibles : 0,27 }\end{array}$ \\
\hline $\begin{array}{l}\text { Île-du-Prince- } \\
\text { Édouard }\end{array}$ & $\begin{array}{c}\text { Moyenne : 81,1 } \\
\text { Médiane : } 83 \\
\text { (El : 72-92) }\end{array}$ & $\begin{array}{c}\text { Moyenne : 47,0 } \\
\text { Médiane : 48 } \\
(\text { El : 40-56) }\end{array}$ & $\begin{array}{c}\text { Moyenne : } 18,3 \\
\text { Médiane : } 19 \\
\text { (El : 11-26) }\end{array}$ & $\begin{array}{l}\text { Moyenne : 7,5 } \\
\text { Médiane : } 7 \\
\text { (El : 5-9) }\end{array}$ & $\begin{array}{c}\text { Moyenne : 4,2 } \\
\text { Médiane : } 4 \\
(\text { El : } 2-6)\end{array}$ & $\begin{array}{c}\text { Moyenne : 1,6 } \\
\text { Médiane : } 1 \\
\text { (El : 0-3) }\end{array}$ & $\begin{array}{l}\text { Existants : } 1,84 \\
\text { Disponibles : } 0,24\end{array}$ \\
\hline Nouvelle-Écosse & $\begin{array}{c}\text { Moyenne : 80,7 } \\
\text { Médiane : } 83 \\
(\text { El : 72-92) }\end{array}$ & $\begin{array}{c}\text { Moyenne : } 47,0 \\
\text { Médiane : } 48 \\
\text { (El : 38-56) }\end{array}$ & $\begin{array}{l}\text { Moyenne : } 18,4 \\
\text { Médiane : } 19 \\
\text { (El : 11-25) }\end{array}$ & $\begin{array}{l}\text { Moyenne : 7,5 } \\
\text { Médiane : } 7 \\
\text { (El:5-9) }\end{array}$ & $\begin{array}{c}\text { Moyenne : 4,2 } \\
\text { Médiane : } 4 \\
(\text { El : } 2-6)\end{array}$ & $\begin{array}{c}\text { Moyenne : } 1,7 \\
\text { Médiane : } 1 \\
\text { (El : } 1-3)\end{array}$ & $\begin{array}{l}\text { Existants : } 1,48 \\
\text { Disponibles : } 0,30\end{array}$ \\
\hline $\begin{array}{l}\text { Terre-Neuve-et- } \\
\text { Labrador }\end{array}$ & $\begin{array}{c}\text { Moyenne : } 81 \\
\text { Médiane : } 83 \\
(\text { El : 73-93) }\end{array}$ & $\begin{array}{c}\text { Moyenne : 46,7 } \\
\text { Médiane : } 47 \\
\text { (El : 40-56) }\end{array}$ & $\begin{array}{l}\text { Moyenne : 18,9 } \\
\text { Médiane : } 19 \\
\text { (El : 12-25) }\end{array}$ & $\begin{array}{l}\text { Moyenne : 7,6 } \\
\text { Médiane : } 7 \\
(\text { El : 5-10) }\end{array}$ & $\begin{array}{c}\text { Moyenne : 4,2 } \\
\text { Médiane : } 4 \\
(\text { EI : 3-6) }\end{array}$ & $\begin{array}{c}\text { Moyenne : } 1,7 \\
\text { Médiane : } 2 \\
\text { (EI : 1-2) }\end{array}$ & $\begin{array}{l}\text { Existants : } 1,85 \\
\text { Disponibles : 0,37 }\end{array}$ \\
\hline
\end{tabular}

Note : COVID-19 = maladie à coronavirus 2019; EI = écart interquartile.

*Les estimations déclarées sont des moyennes et des écarts interquartiles. Pour calculer le nombre de lits de soins intensifs disponibles pour le traitement de patients atteints de la COVID-19, nous avons supposé que le taux d'occupation actuel de ces lits était de $80 \%$. 
Dans le scénario de référence, où $R_{0}=2,5$ et où les cas légers ne s'isolent pas, la demande moyenne pour l'ensemble des provinces serait de 569 (intervalle : 548-587) jours-lits de soins intensifs par 10000 habitants pour la durée de l'épidémie (figure 2), alors estimée à 36 semaines (figures A1 à A4 de l'annexe 1). Selon les projections, c'est en Alberta que les soins intensifs seraient les plus sollicités (moyenne : 587 jours-lits par 10000 habitants; médiane : 589; écart interquartile [EI] : 525657), et à l'île-du-Prince-Édouard qu'ils seraient le moins sollicités (moyenne : 561 jours-lits par 10000 habitants; médiane : 566; El : 497-623) (figure 2). Nous avons estimé qu'il faudrait en moyenne 41 lits de soins intensifs supplémentaires par 10000 habitants dans chaque province pour toute la durée de l'épidémie, ce qui correspond au taux cumulatif de patients qui auront besoin de soins intensifs. Nous prévoyons que le nombre de cas atteindra son sommet dans la première moitié de juin (figure 3), et la prévalence des hospitalisations, entre la mi-juin et la fin juin (figures A1 à A4 de l'annexe 1). Malgré des différences interprovinciales minimes, nous prévoyons que le Manitoba et la ColombieBritannique connaîtront la plus forte demande de lits de soins intensifs par 10000 habitants étant donné leur faible nombre de lits disponibles (tableau 2).

Nous avons ensuite examiné des scénarios dans lesquels des personnes ayant des symptômes légers choisissent de s'isoler, ce qui a pour effet de réduire les hospitalisations et de repousser le sommet de la courbe. Si $R_{0}=2,5$, et que $20 \%$ des cas légers et
$80 \%$ des cas graves s'isolent dans les 24 heures suivant l'apparition des symptômes, l'utilisation globale des lits de soins intensifs diminuerait de $23,6 \%$ à 435 (intervalle : 414-461) jours-lits par 10000 habitants (figure 2). Le nombre moyen de lits supplémentaires nécessaires serait alors de 31 par 10000 habitants, soit $24,4 \%$ de moins qu'en l'absence d'isolement volontaire. Comparativement au scénario de référence, la courbe atteindrait son sommet 4 semaines plus tard au Manitoba, et environ 2 à 3 semaines plus tard dans les autres provinces (figure 3 ), et ce sommet correspondrait à un nombre de cas moindre (figures A1 à A4 de l'annexe 1). Enfin, la demande de lits d'hôpital et de soins intensifs diminuerait respectivement de $42 \%$ et de $43 \%$ à l'échelle nationale (tableau 2).

En faisant passer le taux d'isolement volontaire à $40 \%$, nous avons observé une autre baisse des jours-lits de soins intensifs nécessaires, cette fois de 53,6\%. Par rapport à l'absence d'isolement, la demande se chiffre alors à 264 (intervalle : 250279) jours-lits par 10000 habitants (figure 2), et le sommet est repoussé d'un maximum de 8 semaines (figure 3 ). Dans ce scénario, la première province arrive au sommet de sa courbe épidémique à la mi-juillet, et les provinces de l'Est atteignent ce sommet avant l'Ontario et les provinces de l'Ouest (figure 3). Nos projections prévoient qu'à ce moment, la diminution de la demande de lits d'hôpital et de lits de soins intensifs serait respectivement de $78 \%$ et de $79 \%$. L'épidémie serait prolongée de 12 semaines, et il faudrait en moyenne 19 lits de soins intensifs

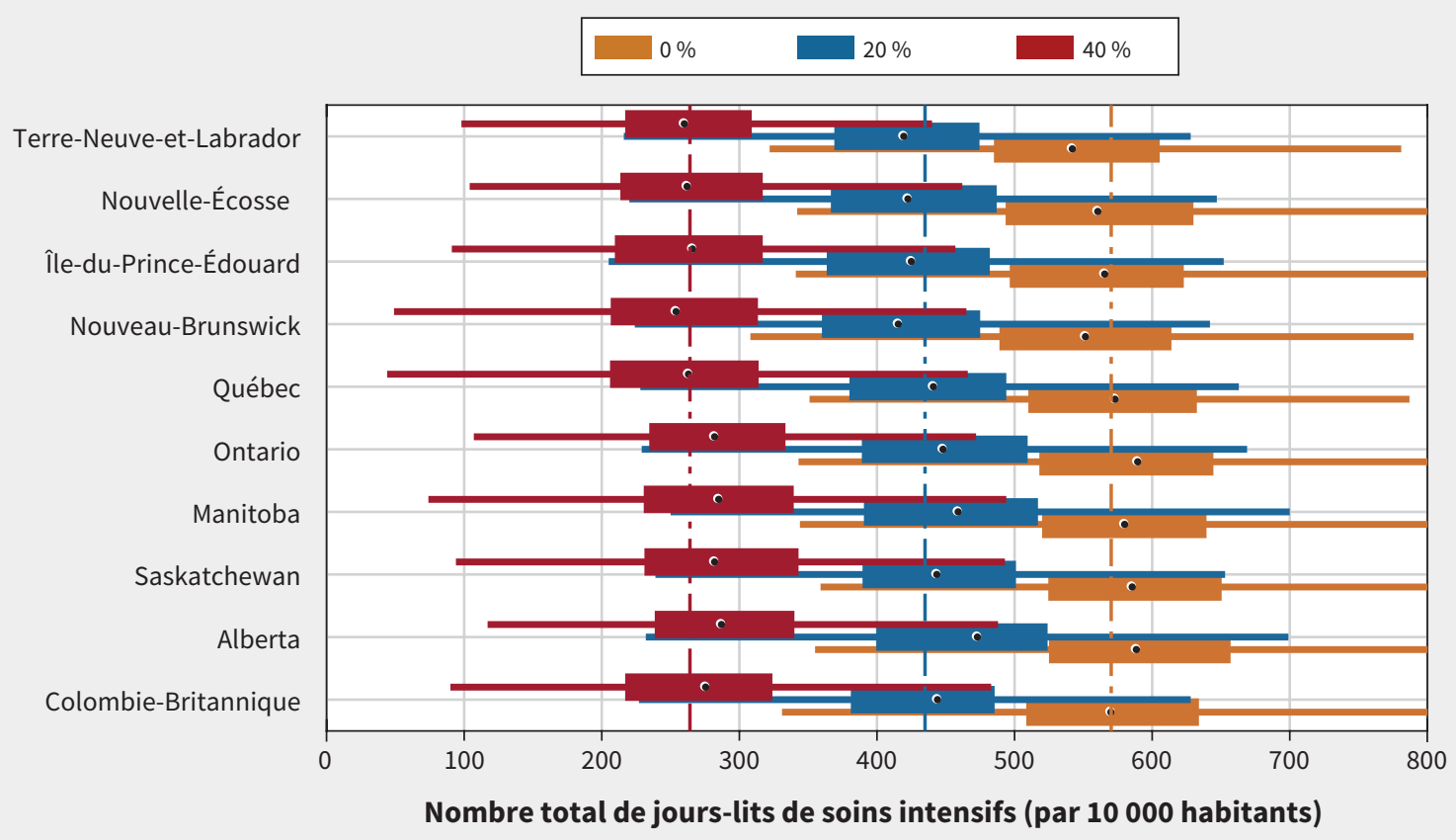

Figure 2. Nombre total de jours-lits de soins intensifs nécessaires par 10000 habitants durant l'épidémie de maladie à coronavirus 2019 (COVID-19), par provinces. Les couleurs indiquent le taux d'isolement volontaire des personnes présentant des symptômes légers : $0 \%$ (en orange), $20 \%$ (en bleu) et $40 \%$ (en rouge). Les cercles à l'intérieur des rectangles représentent la médiane, et les rectangles eux-mêmes, l'écart interquartile (El). Les barres horizontales indiquent l'intervalle étendu, du minimum ( $25^{\mathrm{e}}$ centile $\left.-1,5 \mathrm{EI}\right)$ au maximum $\left(75^{\mathrm{e}}\right.$ centile $\left.+1,5 \mathrm{EI}\right)$. Les traits pointillés verticaux correspondent à la moyenne nationale de jours-lits nécessaires si le taux d'isolement est de $0 \%$ (569; en orange), de $20 \%$ (435; en bleu) et de $40 \%$ ( 264 ; en rouge). Ces distributions sont basées sur les résultats de 500 simulations indépendantes par scénario. 
de plus par 10000 habitants pour toute sa durée, une réduction de 53,7\%. Les résultats de nos calculs avec des taux d'isolement volontaire supérieurs (60\% et $80 \%$ ) sont présentés aux figures A20 à A29 de l'annexe 1 .

En abaissant le $R_{0}$ à 2,0, nous avons obtenu des résultats comparables sur le plan qualitatif, bien que proportionnellement, l'isolement volontaire réduisait beaucoup plus la demande de lits au plus fort de l'épidémie (annexe 1). En supposant que les gens s'isolent 48 heures plutôt que 24 heures après l'apparition des symptômes, nous constatons une augmentation du nombre maximal de cas et des jours-lits de soins intensifs nécessaires (figures A20 à A29 de l'annexe 1).

\section{Interprétation}

Sans vaccin ou traitement modificateur de la maladie scientifiquement éprouvé, l'isolement volontaire des personnes symptomatiques peut ralentir la propagation de la maladie et atténuer la hausse de la capacité d'hospitalisation qu'il faudrait pour absorber les cas de COVID-19. Dans les circonstances plausibles où $R_{0}=2,5$, les résultats révèlent que même si $40 \%$ des patients qui présentent des symptômes s'isolent 24 heures après leur apparition, la demande de lits de soins intensifs à son maximum excédera tout de même la capacité actuelle dans toutes les provinces. Nous prévoyons qu'au sommet de la courbe, le nombre de lits de soins intensifs nécessaires par 10000 habitants (en moyenne) pourrait être jusqu'à 2,6 fois plus élevé que le nombre de lits existants. En appliquant un taux d'occupation réaliste de $80 \%$, nous observons qu'au même moment, la demande pourrait être jusqu'à 13 fois plus élevée que le nombre de lits disponibles, même avec un taux d'isolement volontaire de $40 \%$.

Nous constatons qu'une proportion supérieure de la population devrait s'isoler, afin de limiter la hausse de la pression sur les systèmes de santé provinciaux. Si les ressources sont suffisantes, il faudrait augmenter le nombre de tests de dépistage de la COVID-19. Cette stratégie accroîtrait le pourcentage des cas légers détectés et par le fait même, le taux d'isolement. Sans compter que si l'infection entraîne une immunité, les tests pourraient donner la chance aux personnes rétablies de reprendre leurs activités normales.

\section{Limites de l'étude}

Les résultats doivent être interprétés au regard des hypothèses utilisées dans le modèle. La progression locale de l'épidémie pourrait pousser les autorités à adapter et à étendre leurs mesures contre la maladie, ce qui risque de modifier la courbe de propagation et du même coup nos résultats. Nous n'avons pas expressément modélisé d'autres mesures d'éloignement social, mais nous croyons que la réduction de la transmission par l'isolement volontaire pratiqué à différents taux, susceptible de repousser et d'abaisser le sommet de la courbe et donc la demande de soins intensifs, englobe largement leur effet.

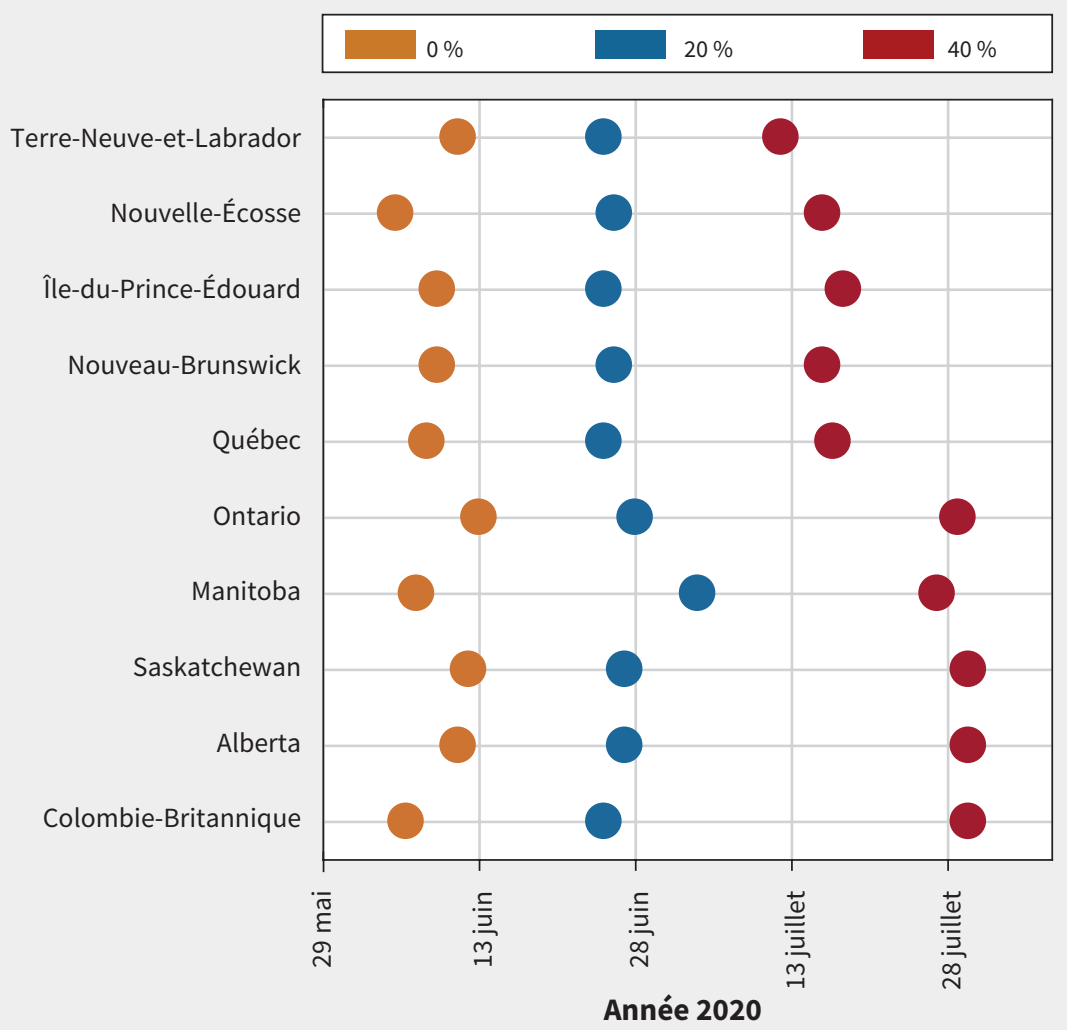

Figure 3. Semaine où l'épidémie devrait être à son plus fort (selon l'incidence quotidienne), par provinces. Les couleurs indiquent le taux d'isolement volontaire des personnes présentant des symptômes légers. 
En outre, dans chaque province, d'autres facteurs d'ordre spatial peuvent repousser l'atteinte de la capacité régionale et de la demande de soins hospitaliers maximales. Par exemple, l'éloignement social (physique) des personnes en santé peut limiter encore davantage la transmission et, ultimement, la charge de morbidité. L'efficacité de l'isolement volontaire varie selon le milieu - rural, urbain, etc. -, surtout pour les groupes vulnérables comme les sans-abri, les personnes au logement ou à l'emploi précaire et les communautés rurales et isolées.

Les territoires servis par différentes autorités de santé publique peuvent aussi se distinguer par la nature des mesures mises en place et la proportion dans laquelle les résidents respectent les directives. Comme nos résultats sont présentés par provinces, ils gomment toute hétérogénéité régionale dans les caractéristiques de la population. Malgré une variabilité régionale ou à plus petite échelle probable, les résultats qualitatifs nationaux peuvent servir dans la prise de décisions stratégiques locales.

Nos projections de l'effet de l'isolement volontaire présupposent que toutes les personnes atteintes développent des symptômes légers ou graves. Étant donné la possibilité de transmission asymptomatique et présymptomatique ${ }^{25}$, l'isolement des cas symptomatiques est probablement moins efficace que souhaité. Nous n'avons pas formellement intégré ces statuts infectieux au modèle, mais en repoussant le délai entre l'apparition des symptômes et l'isolement (48 heures plutôt que 24 heures), nous avons potentiellement tenu compte du risque de contagion par les personnes présymptomatiques.

Nous avons également présumé que les patients hospitalisés faisaient l'objet d'un isolement efficace empêchant toute propagation de l'infection (p. ex., aux professionnels de la santé). Comme les mesures de lutte anti-infectieuse sont rigoureusement suivies par ceux qui traitent les patients atteints de la COVID-19, nous jugeons cette supposition raisonnable dans le milieu canadien des soins hospitaliers actifs. Or des soignants, au Canada comme ailleurs, ont contracté la COVID-19. En présence de transmission communautaire, il est plus probable que les professionnels aient été atteints par ce mode de transmission plutôt qu'à cause d'une faible observance des pratiques de lutte anti-infectieuse dans l'exercice de leurs fonctions. Certains facteurs pouvant nuire au respect des pratiques exemplaires, tels que les pénuries d'équipement de protection individuelle, l'achalandage, la fatigue extrême et le stress, augmentent le risque que les travailleurs de la santé contractent l'infection au travail et la transmettent dans leur milieu de soin, à la maison et dans la communauté jusqu'à son dépistage. L'augmentation du risque serait atténuée par l'application des recommandations visant à lutter contre la maladie, le dépistage précoce auprès du personnel et la baisse du nombre de soignants s'occupant de patients qui n'ont pas la COVID-19 alors que l'épidémie progresse. L'infection de travailleurs de la santé pourrait effectivement réduire l'efficacité de l'isolement en milieu hospitalier, mais pour le moment, la recherche n'a pas quantifié l'effet combiné des facteurs susmentionnés. Cela dit, il pourra être intégré aux modèles à mesure que les données scientifiques s'accumuleront.
Notre modèle ne tient pas compte des facteurs saisonniers, qui peuvent influencer la transmission de la maladie, notamment par un ralentissement estival et une résurgence concomitante à celle de l'influenza à la prochaine saison grippale. Comme ces facteurs ne sont pas quantifiés et qu'on ne connaît pas encore parfaitement les caractéristiques de la COVID-19, les projections quantitatives effectuées avec des modèles demeurent incertaines. Nous pourrons toutefois reparamétrer notre modèle à la lumière des nouvelles connaissances et données sur la COVID-19, et ainsi mieux prévoir l'évolution de la courbe épidémique et la demande de soins intensifs. Enfin, au-delà de ces considérations, les résultats soulignent l'importance des mesures d'éloignement social, et particulièrement de l'isolement volontaire, dans la réduction des besoins en soins hospitaliers et en soins intensifs durant l'épidémie de COVID-19.

\section{Conclusion}

Les résultats soulignent l'importance de l'isolement volontaire dans la réduction de la pression sur les établissements de santé durant l'épidémie de COVID-19, qui s'étalera sur les prochains mois. La capacité relativement faible des soins intensifs au Canada - combinée à des taux d'occupation des lits d'hôpital de plus de $90 \%$ et, dans beaucoup de régions, la quasi atteinte ou le dépassement de la capacité en l'absence de pandémie ${ }^{23}$ - fait ressortir les difficultés que pose le traitement des cas critiques en situation d'épidémie et particulièrement au plus fort de celleci. Dans leur travail de planification, les autorités du pays peuvent s'inspirer de l'expérience des pays qui ont déjà atteint leur sommet de propagation de la COVID-19. Si les scénarios décrits ci-dessus s'avèrent, la planification des soins devra faire place à la gestion de crise, mais à différents moments selon l'endroit. Les provinces et territoires pourraient alors, dans une certaine mesure, se partager des ressources humaines et matérielles. Ils pourraient aussi, tandis qu'ils s'efforcent d'accroître leur capacité à offrir des lits de soins intensifs (ressources humaines, respirateurs, lits, équipement de protection individuelle, aide dans les mesures de lutte anti-infectieuse), utiliser nos projections pour anticiper les besoins, sur les plans temporel et quantitatif. La confirmation en laboratoire du niveau de transmission communautaire donnera une meilleure idée de l'allure de la courbe dans une région. De plus, les gouvernements provinciaux et fédéral ont décidé d'augmenter rapidement le nombre de tests, ce qui facilitera la surveillance de la maladie ${ }^{26-29}$. Selon ce qui a été observé dans d'autres pays, transférer les cas graves aux soins intensifs tôt aide à stabiliser les patients avant qu'ils connaissent une insuffisance respiratoire ${ }^{30,31}$. À noter, pour terminer, que les pratiques exemplaires de prise en charge des cas critiques évoluent rapidement.

\section{Références}

1. Coronavirus COVID-19 global cases by the Center for Systems Science and Engineering (CSSE) at Johns Hopkins University (JHU). Johns Hopkins Coronavirus Resource Center. Accessible ici : www.arcgis.com/apps/opsdashboard/index. html\#/bda7594740fd40299423467b48e9ecf6 (consulté le 19 mars 2020).

2. Ñamendys-Silva SA. Respiratory support for patients with COVID-19 infection. Lancet Respir Med 2020 le 5 mars : pii : S2213-2600(20)30110-7 [Cyberpublication avant impression]. Doi : 10.1016/S2213-2600(20)30110-7. 
3. Wang D, Hu B, Hu C, et al. Clinical characteristics of 138 hospitalized patients with 2019 novel coronavirus-infected pneumonia in Wuhan, China. JAMA 2020 le 7 février [Cyberpublication avant impression]. doi : 10.1001/jama.2020.1585

4. Yang $X, Y u Y, X u$ J, et al. Clinical course and outcomes of critically ill patients with SARS-CoV-2 pneumonia in Wuhan, China: a single-centered, retrospective, observational study. Lancet Respir Med 2020 le 24 février : pii : S2213-2600(20)30079-5 [Cyberpublication avant impression]. Doi : 10.1016/S2213-2600(20)30079-5.

5. Huang C, Wang Y, Li X, et al. Clinical features of patients infected with 2019 novel coronavirus in Wuhan, China. Lancet 2020;395:497-506.

6. Guan W-j, Ni Z-y, Hu Y, et al. Clinical Characteristics of Coronavirus Disease 2019 in China. N Engl J Med 2020 le 28 février [Cyberpublication avant impression]. doi : 10.1056/NEJMoa2002032.

7. Moghadas SM, Shoukat A, Fitzpatrick MC, et al. Projecting hospital utilization during the COVID-19 outbreaks in the United States. Proc Natl Acad Sci U S A 2020 le 3 avril [Cyberpublication avant impression]. Doi : 10.1073/pnas.2004064117.

8. The impact of the H1N1 pandemic on Canadian hospitals. Ottawa: Canadian Institute for Health Information; 2010. Accessible ici : https://secure.cihi.ca/ free_products/H1N1_AIB_final_EN.pdf (consulté le 11 mars 2020).

9. Kumar A, Zarychanski R, Pinto R, et al. Canadian Critical Care Trials Group H1N1 Collaborative. Critically ill patients with 2009 influenza A(H1N1) infection in Canada. JAMA 2009;302:1872-9.

10. Hota S, Fried E, Burry L, et al. Preparing your intensive care unit for the second wave of H1N1 and future surges. Crit Care Med 2010;38(Suppl):e110-119.

11. Eggertson L. Critical care doctors want escalated pandemic planning. CMAJ 2009;181:253-4.

12. Fowler RA, Abdelmalik P, Wood G, et al.; Canadian Critical Care Trials Group. Canadian ICU Capacity Group. Critical care capacity in Canada: results of a national cross-sectional study. Crit Care 2015;19:133.

13. Li Q, Guan X, Wu P, et al. Early transmission dynamics in Wuhan, China, of novel coronavirus-infected pneumonia. N Engl J Med 2020;382:1199-1207.

14. Wu JT, Leung K, Leung GM. Nowcasting and forecasting the potential domestic and international spread of the 2019-nCoV outbreak originating in Wuhan, China: a modelling study. Lancet 2020;395:689-97.

15. Population and demography statistics. Ottawa : Statistique Canada; modifié le 19 mars 2019. Accessible ici : www.statcan.gc.ca/eng/subjects-start/population _and_demography (consulté le 20 mars 2020).

16. Mossong J, Hens N, Jit M, et al. Social contacts and mixing patterns relevant to the spread of infectious diseases. PLoS Med 2008;5:e74.

17. Li R, Pei S, Chen B, et al. Substantial undocumented infection facilitates the rapid dissemination of novel coronavirus (SARS-CoV2). Science 2020 le 16 mars. pii : eabb3221 [Cyberpublication avant impression]. Doi : 10.1126/science. abb3221.

Intérêts concurrents : Joanne Langley signale que l'Université Dalhousie a reçu des fonds de Sanofi, de GlaxoSmithKline, de Merck, de Janssen, de VBI et de Pfizer pour la tenue d'études sur des vaccins. La $D^{\text {re }}$ Langley est titulaire de la chaire en vaccinologie pédiatrique des Instituts de recherche en santé du Canada et de GlaxoSmithKline. Aucun autre intérêt concurrent déclaré.

Cet article a été révisé par des pairs.

Affiliations : Center for Infectious Disease Modeling and Analysis (A. Shoukat, C. Wells, A. Galvani), École de santé publique de Yale, New Haven (Connecticut); Centre canadien de vaccinologie (J. Langley), Université Dalhousie, Centre de soins de santé IWK et Régie de la santé de la Nouvelle-Écosse (J. Langley), Halifax (Nouvelle-Écosse); Emerging Pathogens Institute (B. Singer), Université de Floride, Gainesville (Floride); Agent-Based Modelling Laboratory (S. Moghadas), Université York, Toronto (Ontario).

Collaborateurs : Affan Shoukat, Alison Galvani et Seyed Moghadas ont contribué à la conception et à l'organisation du travail. Affan Shoukat, Chad Wells, Joanne Langley et Seyed Moghadas ont contribué à
18. Lauer SA, Grantz $\mathrm{KH}, \mathrm{Bi} \mathrm{Q}$, et al. The incubation period of coronavirus disease 2019 (COVID-19) from publicly reported confirmed cases: estimation and application. Ann Intern Med 2020 le 10 mars [Cyberpublication avant impression]. Doi : 10.7326/M20-0504.

19. Report of the WHO-China joint mission on coronavirus disease 2019 (COVID-19). Genève : Organisation mondiale de la santé. Du 16 au 24 février 2020. Accessible ici : www.who.int/docs/default-source/coronaviruse/who-china-joint-mission-on -covid-19-final-report.pdf (consulté le 9 mars 2020).

20. Sanche S, Lin YT, Xu C, et al. The novel coronavirus, 2019-nCoV, is highly contagious and more infectious than initially estimated. medRxiv 2020 le 11 février [Cyberpublication avant impression]. Doi : 10.1101/2020.02.07.20021154.

21. MIDAS Network. MIDAS 2019 novel coronavirus repository. San Francisco: GitHub. Accessible ici : https://github.com/midas-network/COVID-19 (consulté le 27 février 2020).

22. Loustalot F, Silk BJ, Gaither A, et al. Household transmission of 2009 Pandemic influenza $A(\mathrm{H} 1 \mathrm{~N} 1)$ and nonpharmaceutical interventions among households of high school students in San Antonio, Texas. Clin Infect Dis 2011;52(Suppl 1):S146-153.

23. OECD. Hospital beds. In: Health at a Glance 2017: OECD Indicators. OECD (Organisation for Economic Co-operation and Development) Publishing, Paris, France; 2017:172-3.

24. Bezanson J, Edelman A, Karpinski S, et al. Julia: a fresh approach to numerical computing. SIAM Rev 2017;59:65-98.

25. Du Z, Xu X, Wu Y, et al. Serial interval of COVID-19 from publicly reported confirmed cases. Emerg Infect Dis 2020 le 19 mars [Cyberpublication avant impression]. Doi : 10.3201/eid2606.200357.

26. Province announces new measures to fight the coronavirus. 104.7 Heart FM 2020 le 12 mars. Accessible ici : www.heartfm.ca/news/local-news/province -announces-new-measures-to-fight-the-coronavirus/ (consulté le 19 mars 2020).

27. Jones RP, Tunney C. Government expanding COVID-19 testing capacity and purchase of medical equipment, says federal health minister. CBC News 2020 le 18 mars, mis à jour 2020 le 19 mars. Accessible ici : www.cbc.ca/news/politics/ coronavirus-test-capacity-medical-supplies-1.5500662 (consulté le 19 mars 2020).

28. Wong J. Coronavirus: Alberta plans for expanded testing capacity and assessment centres. Global News 2020 le 7 mars. Accessible ici : https://globalnews.ca/ news/6645687/alberta-coronavirus-testing-assessment-centres/ (consulté le 19 mars 2020).

29. Zussman RBC. Premier outlines province's COVID-19 response plan. Global News 2020 le 6 mars. Accessible ici : www.//globalnews.ca/news/6641644/b-c -coronavirus-covid-19-update/ (consulté le 19 mars 2020).

30. Murthy S, Gomersall CD, Fowler RA. Care for critically ill patients with COVID-19. JAMA 2020 le 11 mars [Cyberpublication avant impression]. Doi : 10.1001/jama.2020.3633.

31. Liew MF, Siow WT, MacLaren G, et al. Preparing for COVID-19: early experience from an intensive care unit in Singapore. Crit Care 2020;24:83. l'acquisition, à l'analyse et à l'interprétation des données. Tous les auteurs ont participé à la rédaction du manuscrit, en ont révisé de façon critique le contenu intellectuel important, ont donné leur approbation finale pour la version destinée à être publiée, et assument l'entière responsabilité de tous les aspects du travail.

Financement : Alison Galvani : National Institutes of Health (UO1GM087719, 1RO1Al151176-01); Burnett \& Stender Families Endowment; Fondation Notsew Orm Sands. Seyed Moghadas : IRSC (OV4 - 170643), intervention de recherche rapide contre la maladie à coronavirus 2019 (COVID-19); Conseil de recherches en sciences naturelles et en génie du Canada); Fondation canadienne pour l'innovation.

Transmission des données : Un modèle informatique avec des valeurs de paramètres et des données relatives à la simulation est accessible gratuitement à l'adresse https://github.com/affans/covid19abm.jl.

Accepté : 2 avril 2020.

Correspondance : Joanne Langley, Joanne.Langley@dal.ca 